\title{
Fragilidad y estado nutricional en el periodo pre-trasplante. ¿Existe relación?
}

\section{Maria Vera Casanova, Anna Bach Pascual, Aida Martínez Sáez, Yolanda Castillo Piña, Anna Faura Vendrell, Dolores Redondo Pachón}

Servicio de Nefrología. Hospital del Mar. Institut Mar d’Investigacions Mèdiques. Barcelona. España

\section{Resumen}

Introducción: La malnutrición en la enfermedad renal crónica (ERC) está asociada al aumento del riesgo de mortalidad y complicaciones. Sin embargo, el estado nutricional de los pacientes con ERC en lista de espera (LE) de trasplante renal (TR), y su relación con el estado de fragilidad ha sido poco evaluado.

Objetivo: Analizar el estado nutricional y funcional de una cohorte de pacientes en lista de espera de trasplante renal y su relación con la fragilidad.

Pacientes y Método: Estudio retrospectivo de los pacientes incluidos en LE de TR desde Junio 2016 hasta Junio 2017. Se evaluaron a los pacientes mediante distintas escalas de valoración, recogiendo parámetros antropométricos, analíticos y de bioimpedanciometría (BCM).

Resultados: De los 177 pacientes incluidos, 55 $(31.1 \%)$ se definieron como frágiles. Dicho grupo eran de mayor edad (64.2 vs 61.2 años; $p=0.08$ ), sexo femenino ( $56.4 \%$ vs $32.8 \%, p<0.01$ ), IMC mayor $\left(29.3 \pm 5.8\right.$ vs $\left.27.3 \pm 5,6 \mathrm{~kg} / \mathrm{m}^{2} ; p=0.03\right)$ y más frecuentemente diabéticos (DM2) (43.6\% vs $30.3 \%$; $\mathrm{p}=0.08)$. La evaluación mediante $\mathrm{BCM}$ demostró que los pacientes frágiles tenían menos índice de tejido magro $\left(12.3 \pm 2.4\right.$ vs $\left.14.1 \pm 4 \mathrm{~kg} / \mathrm{m}^{2} ; p<0.01\right)$ y mayor

Anna Bach Pascual

Servicio de Nefrología. Hospital del Mar

Institut Mar d'Investigacions Mèdiques. Barcelona

Passeig Marítim 25-29. 08003 Barcelona

E-mail: abach@parcdesalutmar.cat índice de tejido graso $\left(16.2 \pm 6.1\right.$ vs $11.8 \pm 5.6 \mathrm{~kg} / \mathrm{m}^{2}$; $p<0.01$ ) que los no frágiles.

Conclusión: En nuestra lista de espera de TR, los pacientes frágiles son de mayor edad, sexo femenino, mayor grado de obesidad, DMII y con menor masa muscular. Las estrategias encaminadas a liberalizar dietas, aumentar la ingesta proteica y fomentar el ejercicio físico pueden ser relevantes para mejorar los resultados a corto y largo plazo del TR.

PALABRAS CLAVE: trasplante renal; fragilidad; malnutrición; bioimpedanciometría; dependencia.

Frailty and nutritional status in the pre-transplant period. Is there a relationship?

\section{Abstract}

Introduction: Malnutrition in chronic kidney disease (CKD) is associated with increased risk of mortality and complications. However, the nutritional status of patients with CKD on the renal transplant waiting list and their relationship to the frailty status has been poorly evaluated.

Aim: To analyze the nutritional and functional status of a cohort of patients on the waiting list for renal transplantation and its relation to frailty.

Patients and method: Retrospective study of patients included in waiting list of renal trasplantation from June 2016 to June 2017. Patients were evaluated through different assessment scales, taking anthropometric, analytical and bioimpedancometry (BCM) parameters. 
Results: Of the 177 patients included, 55 (31.1\%) were defined as frailty. This frailty group was older (64.2 vs 61.2 years, $p=0.08$ ), higher number of females $(56.4 \%$ vs $32.8 \%, p<0.01)$, higher BMI $\left(29.3 \pm 5.8\right.$ vs $\left.27.3 \pm 5.6 \mathrm{~kg} / \mathrm{m}^{2}, \mathrm{p}=0.03\right)$ and more frequently diabetic (DMII) $(43.6 \%$ vs $30.3 \%$, p $=0.08$ ). The BCM evaluation showed that the frailty patients had a lower index of lean tissue $(12.3 \pm 2.4$ vs $14.1 \pm 4 \mathrm{~kg} / \mathrm{m}^{2}, \mathrm{p}<0.01$ ) and a higher fat index ( $16.2 \pm 6.1$ vs $\left.11.8 \pm 5.6 \mathrm{~kg} / \mathrm{m}^{2} ;<0.01\right)$ than nonfrailty ones.

Conclusions: In our waiting list for renal transplantation, the frailty patients are older, female, more obese, DMII and with lower muscle mass. Strategies to liberalize diets, increase protein intake and promote physical exercise may be relevant to improve the short- and long-term results of the renal transplantation.

KEYWORDS: renal transplantation; frailty; malnutrition; bioimpedanciometry; dependence.

\section{Introducción}

En las últimas décadas los avances en medicina y el hecho de tener una población más envejecida han hecho que la prevalencia de la enfermedad renal crónica (ERC) vaya en aumento ${ }^{1}$. A su vez, esto está provocando que los pacientes que requieren de terapia renal sustitutiva, ya sea en forma de diálisis o de trasplante renal (TR), sean también de mayor edad.

Como consecuencia de estos cambios, el perfil de pacientes en lista de espera ( $L E$ ) para un $T R$, crecen no solo en edad, sino que también en comorbilidades y por lo tanto, aumentan los riesgos de sufrir más complicaciones peri y post cirugía.

La prevalencia de ERC que requiere terapia sustitutiva en España es de 1211,5 ppm; un $23 \%$ de estos pacientes tienen entre 65 y 74 años y un $20 \% \geq 75$ años. Aproximadamente 4.300 pacientes están incluidos en lista de espera para recibir un trasplante renal ${ }^{1}$.

La fragilidad es la falta de reservas biológicas en un individuo, que frente a un proceso de estrés aumenta el riesgo de complicaciones, reingresos e incluso de mortalidad. Según la OMS, la malnutrición se define como el conjunto de carencias, excesos o desequilibrios de la ingesta de energía y/o nutrientes de una persona.
Por un lado puede distinguirse la desnutrición, el déficit ponderal y de crecimiento, así como el déficit de minerales y micronutrientes y por otro lado el sobrepeso, la obesidad y las enfermedades no transmisibles relacionadas con el régimen alimentario, como la diabetes mellitus tipo $\mathrm{II}^{2}$.

La nutrición es un factor estrechamente relacionado con la fragilidad ${ }^{3}$, dado que todos los criterios pueden verse alterados frente a unos hábitos pobres de alimentación. Está descrito que por diferentes motivos, entre un $30 \%$ y un $70 \%$ de pacientes en diálisis pueden presentar malnutrición ${ }^{4}$.

Teniendo presentes los datos descritos anteriormente, nos plantemos analizar cómo son los pacientes con ERC avanzada incluidos en nuestra lista de espera de TR en cuanto a dos áreas de evaluación clínica muy relevantes, como la fragilidad y la nutrición, y la posible interacción entre ambas.

\section{Objetivo}

Analizar el estado nutricional y funcional de una cohorte de pacientes en lista de espera de trasplante renal y su relación con la fragilidad.

\section{Pacientes y Método}

Estudio observacional y retrospectivo. Se analizaron los datos obtenidos mediante práctica clínica habitual, de los pacientes visitados e incluidos en lista de espera de nuestro servicio para TR, desde Junio de 2016 hasta Junio de 2017.

\section{Criterios de inclusión}

Se incluyeron todos los pacientes que optaban a un TR y que fueron evaluados por la enfermera referente de TR antes del acto quirúrgico.

\section{Criterios de exclusión}

Excluimos aquellos pacientes a los cuales no se les pudo hacer la valoración antes del TR ya sea por barrera idiomática, déficit cognitivo o TR previo a la valoración.

\section{Variables a estudio}

Las variables a estudio se recogieron en el momento de inclusión en lista de espera, en el curso de la primera valoración por parte de enfermería. 
- Datos demográficos: Fecha de nacimiento, sexo y raza.

- Datos personales: Nivel de conocimiento del idioma, nivel de estudios, nivel socioeconómico y soporte familiar.

- Datos relacionados con ERC: Tipo de terapia renal sustitutiva y fecha de inicio, fecha de inclusión en lista de espera, causa de la enfermedad renal, tipo de donante y fecha del TR.

- Diagnóstico de diabetes mellitus tipo II (DM).

- Datos antropográficos: Peso, talla, índice de masa corporal (IMC) y peso seco en diálisis.

- Datos de la bioimpedanciometría (BCM): estado de hidratación, porcentaje de masa muscular y masa grasa.

- Variables analíticas: Creatinina, urea, sodio, potasio, calcio, fosforo, PTH, Vitamina D (OH25), albúmina sérica, proteínas totales, proteína $C$ reactiva, colesterol total, triglicéridos, colesterol HDL, colesterol LDL, hemoglobina, hematocrito, volumen corpuscular medio, ferritina, saturación de transferrina, hierro, vitamina B12, ácido fólico, hemoglobina glicosilada.

- Estudio de la fragilidad: utilizamos el test definido y validado por Fried ${ }^{5}$ que evalúa cinco criterios:

1. Pérdida involuntaria de peso (Shrinking).

2. Baja energía o agotamiento (Exhaustion).

3. Lentitud en la marcha (Slowness).

4. Debilidad muscular (Weakness).

5. Baja actividad física (Low physical activity).

- Otras escalas de valoración:

1. Índice de Barthel: Evalúa el grado de dependencia para las actividades de la vida diaria6.

2. Test de Lawton y Brody: Evalúa el nivel de dependencia para las actividades instrumentales de la vida diaria ${ }^{7}$.

3. Short Nutritional Assessment Questionnaire (SNAQ), que predice el riesgo de malnutrición.

4. Test Morinsky Green: Evalúa la adherencia al tratamiento ${ }^{8}$.

5. Test Yesavage: Evalúa el riesgo de padecer una depresión?.

6. Test que evalúa el riesgo de sufrir exclusión social.

Todos los test fueron realizados por los propios pacientes sin sugestión o influencias externas.

\section{Aspectos éticos}

El estudio se llevó a cabo siguiendo las normas dictadas por la Declaración de Helsinki, las Normas de Buena Práctica Clínica y cumpliendo la legislación vigente. EI tratamiento de los datos de carácter personal de los sujetos participantes en el estudio, se ajustó a lo establecido en la Ley Orgánica de Protección de Datos de Carácter Personal, 15/1999 del 13 de Diciembre y a la Directiva Europea sobre Privacidad de Datos.

\section{Aspectos estadísticos}

Los datos se expresaron con medias y desviación estándar, con mediana y rango intercuartílico o con frecuencias y porcentajes. Se utilizó el test de chi-cuadrado para comparar variables cualitativas; y $\mathrm{T}$ de student para comparar variables cuantitativas con distribución normal. Si no seguían una distribución normal, se utilizó el test U de Mann Whitney. Se estableció el nivel de significación estadística en $p<0.05$. Para hacer el análisis estadístico utilizamos el programa SPSS versión 22.

\section{Resultados}

\section{Pacientes}

Se incluyeron en el estudio 177 pacientes, 71 (40.1\%) mujeres, con una edad media de $62,1 \pm 10,4$ años y un IMC de $27,9 \pm 5,7 \mathrm{Kg} / \mathrm{m}^{2}$. Sesenta y uno $(34.5 \%)$ eran diabéticos. Los pacientes llevaban un tiempo medio en diálisis de $30.1 \pm 26,4$ meses y un tiempo en lista de espera de $16,6 \pm 12,7$ meses.

Aplicando el test de Fried, se clasificó a los pacientes en tres grupos: frágiles $(n=14)$, frágiles leves $(n=41)$ y no frágiles $(n=122)$, agrupándose los frágiles y frágiles leves para realizar el análisis (figura $\mathbf{1}$ ).

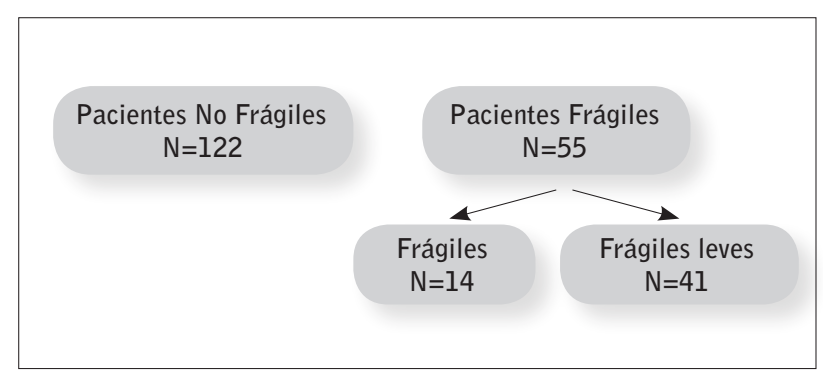

Figura 1. Flujograma de pacientes. 
Tabla 1. Características generales de la población a estudio.

\begin{tabular}{|c|c|c|c|}
\hline & $\begin{array}{l}\text { Pacientes Frágiles } \\
\qquad(\mathrm{N}=55)\end{array}$ & $\begin{array}{c}\text { Pacientes no Frágiles } \\
(\mathrm{N}=122)\end{array}$ & p \\
\hline Edad (años) [media (DE)] & $64.17(10.2)$ & $61.22(10.4)$ & 0.08 \\
\hline Raza caucásica $(n, \%)$ & $54(98.2)$ & $118(96.7)$ & 0.58 \\
\hline Sexo, Mujeres ( $n, \%)$ & $31(56.4)$ & $40(32.8)$ & $<0.01$ \\
\hline IMC kg7m2 [media (DE)] & $29.35(5.8)$ & $27.31(5.6)$ & 0.03 \\
\hline Diabetes tipo II $(n, \%)$ & $24(43.6)$ & $37(30.3)$ & 0.08 \\
\hline Tipo de diálisis, HD (n, \%) & $42(77.8)$ & $77(63.3)$ & 0.13 \\
\hline $\begin{array}{l}\text { Tiempo en diálisis (meses) } \\
\text { [mediana (RIQ)] }\end{array}$ & $28(14.2-42)$ & $23(4.2-13.2)$ & 0.02 \\
\hline $\begin{array}{l}\text { Tiempo en lista de espera (meses) } \\
\text { [mediana (RIQ)] }\end{array}$ & $15(6-24)$ & $14.5(5-23.7)$ & 0.91 \\
\hline \multicolumn{4}{|l|}{$\begin{array}{l}\text { Características funcionales } \\
\text { y riesgo de depresión }\end{array}$} \\
\hline Índice de Barthel (90-61) (n, \%) & $7(13.7)$ & $3(2.5)$ & $<0.01$ \\
\hline Test de Lawton y Brody $\leq 4(n, \%)$ & $10(19.6)$ & $7(5.8)$ & $<0.01$ \\
\hline Test Yesavage positivo $(n, \%)$ & $30(66.7)$ & $61(55.5)$ & 0.2 \\
\hline \multicolumn{4}{|l|}{ Datos socioeconómicos } \\
\hline Bajo conocimiento del idioma $(n, \%)$ & $6(10.9)$ & $10(8.2)$ & 0.58 \\
\hline Nivel de estudios ( $n, \%)$ & $37(68.5)$ & $71(62.8)$ & 0.47 \\
\hline Soporte familiar deficiente $(n, \%)$ & $12(32.4)$ & $10(12.5)$ & $<0.01$ \\
\hline
\end{tabular}

Tabla 2a. Características nutricionales de la población a estudio.

\begin{tabular}{lccc} 
& $\begin{array}{c}\text { Pacientes Frágiles } \\
(\mathrm{N}=55)\end{array}$ & $\begin{array}{c}\text { Pacientes no Frágiles } \\
(\mathrm{N}=122)\end{array}$ & $\mathbf{p}$ \\
\hline Albúmina [media (DE)] & $4.27(0.35)$ & $4.26(0.5)$ & 0.54 \\
\hline Proteínas totales [media (DE)] & $7.07(0.6)$ & $8.12(8.5)$ & 0.53 \\
\hline Colesterol total [media (DE)] & $150.88(32.6)$ & $159.02(37.59)$ & 0.59 \\
\hline PCR [media (DE)] & $1.28(2.7)$ & $2.89(1.2)$ & 0.22 \\
\hline Hemoglobina [media (DE)] & $12.09(1.4)$ & $12.23(1.5)$ & 0.54 \\
\hline Hematocrito [media (DE)] & $36.22(8.2)$ & $37.87(5.2)$ & 0.31 \\
\hline $\begin{array}{l}\text { Saturación transferrina } \\
\text { [media (DE)] }\end{array}$ & $30.31(16.3)$ & $29.77(13.1)$ & 0.84 \\
\hline $\begin{array}{l}\text { Hemoglobina glicosilada } \\
\text { [media (DE)] }\end{array}$ & $6.36(1.5)$ & $6.69(5.8)$ & 0.66 \\
\hline PTH [mediana (RIQ)] & $290.5(204.7-663)$ & $263.5(138.2-410.5)$ & 0.04 \\
\hline Vitamina D25 [mediana (RIQ)] & $10(6-15.7)$ & $10(7-18)$ & 0.19 \\
\hline Ácido Fólico [mediana (RIQ)] & $4.8(2.5-11)$ & $5(2.7-7.9)$ & 0.50 \\
\hline Vitamina B12 [mediana (RIQ)] & $510.5(373.5-572.7)$ & $496(401.7-625.2)$ & 0.73 \\
\hline
\end{tabular}

En cuanto al grado funcional vemos que los pacientes frágiles presentan mayor grado de dependencia que los no frágiles, teniendo un Índice de Barthel y el Test de Lawton y Brody con peores puntuaciones.

Los pacientes más vulnerables (frágiles) tienen peor soporte familiar.

No se detectaron diferencias significativas respecto a la raza, tipo de diálisis, tiempo en espera de TR, riesgo de padecer depresión, conocimiento del idioma o nivel de estudios (tabla 1).

\section{Frágiles vs no frágiles: descripción del estado nutricional}

Analizando el perfil nutricional de los pacientes no encontramos diferencias significativas en relación a los parámetros analíticos evaluados exceptuando la PTH (tabla 2a).

Se observaron diferencias significativas en las medidas obtenidas de la BCM: los pacientes frágiles tienen mayor porcentaje de masa grasa y menor porcentaje de masa muscular, sin diferencias en el estado de hidratación ni en el test de Riesgo de Malnutrición (SNAQ). (tabla 2b).

\section{Factores de riesgo de fragilidad (análisis multivariante)}

Después de realizar un análisis multivariante ajustado, los pacientes frágiles presentaron con más frecuencia un Lawton-Brody inferior a 5 (OR 7,8 [IC 95\% 1.2-48.5], $p=0.026$ ), y presentaron un mayor porcentaje de masa grasa [OR 1.5 [1.01-2.32], $\mathrm{p}=0.04$ ) (tabla 3).

\section{Frágiles vs no frágiles: descripción general}

Cuando comparamos los dos grupos observamos que los pacientes con algún grado de fragilidad tienen mayor edad, mayoritariamente son mujeres con un IMC mayor y con más prevalencia de DM. Los pacientes frágiles permanecen más tiempo en diálisis que el otro grupo.

\section{Discusión y Conclusiones}

En estudios previos se ha demostrado que los pacientes de edad avanzada se benefician del TR en lugar de seguir en diálisis, aunque el aumento de supervivencia disminuye cuando el paciente supera la edad de 70 años $^{5,6}$. El beneficio del TR y el creciente envejecimiento de los 
Tabla 2b. Composición corporal y riesgo de malnutrición de la población a estudio.

\begin{tabular}{|c|c|c|c|}
\hline & $\begin{array}{l}\text { Pacientes Frágiles } \\
\qquad(\mathrm{N}=55)\end{array}$ & $\begin{array}{l}\text { Pacientes no Frágiles } \\
\text { ( } N=122)\end{array}$ & p \\
\hline Hidratación (OH) [media (DE)] & $1.02(1.48)$ & $1.14(1.51)$ & 0.48 \\
\hline $\begin{array}{l}\text { Índice de tejido graso (FTI) } \\
\text { [media (DE)] }\end{array}$ & $16.18(6.08)$ & $11.80(5.59)$ & $<0.01$ \\
\hline $\begin{array}{l}\text { Índice de tejido magro (LTI) } \\
\text { [media (DE)] }\end{array}$ & $12.32(2.49)$ & $14.13(4.03)$ & 0.01 \\
\hline $\begin{array}{l}\text { Short Nutritional Assessment } \\
\text { Questionnaire (SNAQ) }<14(n, \%)\end{array}$ & $15(28.13)$ & $27(22.7)$ & 0.42 \\
\hline
\end{tabular}

Tabla 3. Análisis multivariante del riesgo de fragilidad de nuestra población.

\begin{tabular}{lccc}
\hline & $\begin{array}{c}\text { Odds Ratio } \\
\text { de Confianza }\end{array}$ & 95\% Intervalo & $\mathbf{p}$ \\
\hline Sexo & 0.411 & $0.105-1.606$ & 0.201 \\
\hline IMC & 0.734 & $0.734-0.474$ & 0.165 \\
\hline Tiempo TRS & 0.988 & $0.960-1.016$ & 0.401 \\
\hline Barthel <100 & 2.789 & $0.452-17.197$ & 0.269 \\
\hline Lawton Brody <5 & 7.895 & $1.283-48.590$ & $\mathbf{0 . 0 2 6}$ \\
\hline Soporte familiar bajo & 2.916 & $0.603-14.101$ & 0.183 \\
\hline Índice de tejido magro & 1.164 & $0.833-1.626$ & 0.373 \\
\hline Índice de tejido graso & 1.531 & $1.010-2.321$ & $\mathbf{0 . 0 4 5}$ \\
\hline
\end{tabular}

pacientes en diálisis han provocado la expansión de los criterios para incluir a los pacientes en lista de espera de TR. Como consecuencia, el perfil de los pacientes candidatos a someterse a un TR es más complejo, y en muchos casos se asocia con una situación de fragilidad.

McAdams-Demarco ya demostró en su estudio de 537 pacientes, que la fragilidad tiene un alto impacto sobre la supervivencia del paciente con injerto renal ${ }^{7}$. Nuestro grupo se planteó la necesidad de estudiar y analizar el perfil de nuestros pacientes en lista de espera de TR, en el que encontramos que el $31,1 \%$ presentaban algún grado de fragilidad. El perfil del paciente frágil en nuestra lista de espera para TR es una mujer de mediana edad con sobrepeso, con diabetes mellitus, más de dos años en diálisis, con poco apoyo familiar y grado moderado de dependencia para las actividades de la vida diaria.

Está descrito que en función de los parámetros estudiados existe entre un 30-70\% de malnutrición en pacientes en diálisis ${ }^{4}$. La malnutrición en estos pacientes es multicausal, siendo el principal factor desencadenante la baja ingesta proteica. La ingesta de proteínas está estrechamente relacionada con la presencia de masa muscular y la fuerza, ambos factores directamente involucrados en el desarrollo de la fragilidad. Al analizar los resultados de la BCM como herramienta para poder conocer la composición corporal de nuestros pacientes, podemos observar que los pacientes frágiles tienen menor masa muscular a pesar de que analíticamente no pudimos confirmar el déficit de proteínas, albumina sérica ni micronutrientes ${ }^{3,8}$.

Una vez observados y analizados todos los resultados obtenidos afirmamos que nuestros pacientes no están desnutridos, sino que según la definición de la OMS, están malnutridos. La composición corporal de los pacientes frágiles esta descompensada, presentando mayor masa grasa y menor masa muscular respecto a la normalidad y aunque analíticamente no se observan signos de desnutrición probablemente la ingesta calórica no sea equilibrada. Sería muy recomendable cambiar las dietas de estos pacientes, aumentando la ingesta proteica y fomentar una mayor actividad física a través de pautas consensuadas con los especialistas (fisioterapeutas) individualizadas y adaptadas a la situación de cada paciente.

En conclusión la malnutrición en forma de masa grasa aumentada junto con el grado de dependencia alto, son factores de riesgo independientes asociados con la presencia de fragilidad. Las enfermeras, como profesionales de referencia en la mejora y mantenimiento de hábitos saludables, pueden jugar un papel muy importante para prevenir y/o mejorar la fragilidad de los pacientes en lista de espera para TR. De este modo sería posible minimizar la morbilidad y los resultados posteriores.

\section{Agradecimientos}

Las autoras agradecen a María José Pérez, Ernestina Junyent, Julio Pascual y a todo el equipo de Nefrología por su inestimable colaboración en este proyecto. 
Recibido: 15 abril 2017

Revisado: 27 mayo 2017

Modificado: 12 agosto 2017

Aceptado: 20 agosto 2017

\section{Bibliografía}

1. Registro Español de Enfermos Renales. Informe de Diálisis y Trasplante 2015 (REER). 2016.

2. Oraganización Mundial de la Salud ¿Que es la malnutrición?. [Consultado 2 Marzo 2017]. Disponible en: http://www.who.int/features/qa/malnutrition/es/

3. Yannakoulia M, Ntanasi E, Anastasiou CA, Scarmeas N. Running head: Frailty and nutrition: from epidemiological and clinical evidence to potencial mechanism. Metabolism. Elsevier B.V.; 2016. [Consultado 16 Marzo 2017]. Disponible en: http://dx.doi.org/10.1016/j.metabol.2016.12.005

4. Riobó P, Ortiz A. Nutrición en la Insuficiencia renal. Nutri Info5. 2017. [Consultado 12 julio 2017]. Disponible en: http://www.fresenius-kabi.es/pdf/ nutri_info/Nutri_Info_05.pd

5. Fried LP, Tangen CM, Walston J, Newman AB, Hirsch C, Gottdiener J, et al. Frailty in older adults: evidence for a phenotype. J Gerontol A Biol Sci Med Sci. 2001;56(3):M146-56.

6. Mahoney F, Barthel D. Functional evaluation: The Barthel index. Md State Med J. 1965;14:56-61.

7. Lawton MP, Brody EM. Assessment of Older People: Self-Maintaining and Instrumental Activities of Daily Living. The Gerontologist. 1979 0ct; 9,(3):179-186,. Disponible en: http://dx.doi. org/10.1093/geront/9.3_Part_1.179

8. Solán XN, Redó MLS, García JV, Recerca U De, Urfoa A, Medicina S De. Instrumentos de medida de adherencia al tratamiento. 2007;24:138-41.
9. Martínez de la Iglesia J., Onís Vilches $M^{a}$ C., Dueñas Herrero R., Albert Colomer C., Aguado Taberné C., Luque Luque R.. Versión española del cuestionario de Yesavage abreviado (GDS) para el despistaje de depresión en mayores de 65 años: adaptación y validación. Medifam. 2002 Dic; 12( 10 ): 26-40. [Consultado 27 septiembre 2017]. Disponible en: http://scielo. isciii.es/scielo.php?script=sci_arttext\&pid=S1131$57682002001000003 \& \operatorname{lng}=$ es.

10. Lloveras J, Arcos E, Comas J, Crespo M, Pascual J.A Paired Survival Analysis Comparing Hemodialysis and Kidney Transplantation From Deceased Elderly Donors Older Than 65 Years. Transplantation. 2015;99(5):991-6.

11. Pérez-Sáez MJ, Arcos E, Comas J, Crespo M, Lloveras J, Pascual J. Survival Benefit From Kidney Transplantation Using Kidneys From Deceased Donors Aged $\geq 75$ Years: A Time-Dependent Analysis. Am J Transplant. Sep 2016: 16(9):272433. [Consultado 7 mayo 2017]. Disponible en: http://doi.wiley.com/10.1111/ajt.13800

12. McAdams-Demarco MA, Law A, King E, Orandi B, Salter M, Gupta N, et al. Frailty and mortality in kidney transplant recipients. Am J Transplant. 2015;15(1):149-54.

13. Lukaski HC, Kyle UG, Kondrup J. Assessment of adult malnutrition and prognosis with bioelectrical impedance analysis: phase angle and impedance ratio. Curr Opin Clin Nutr Metab Care. 2017;1-10. 